\title{
Univ.-Prof. Dr. phil. Dr. H. c. mult. Hellmut Fischmeister am 6. November 2019 verstorben
}

\section{Bruno Hribernik}

ASMET, A 8700 Leoben, Österreich

Online publiziert 12. Dezember 2019

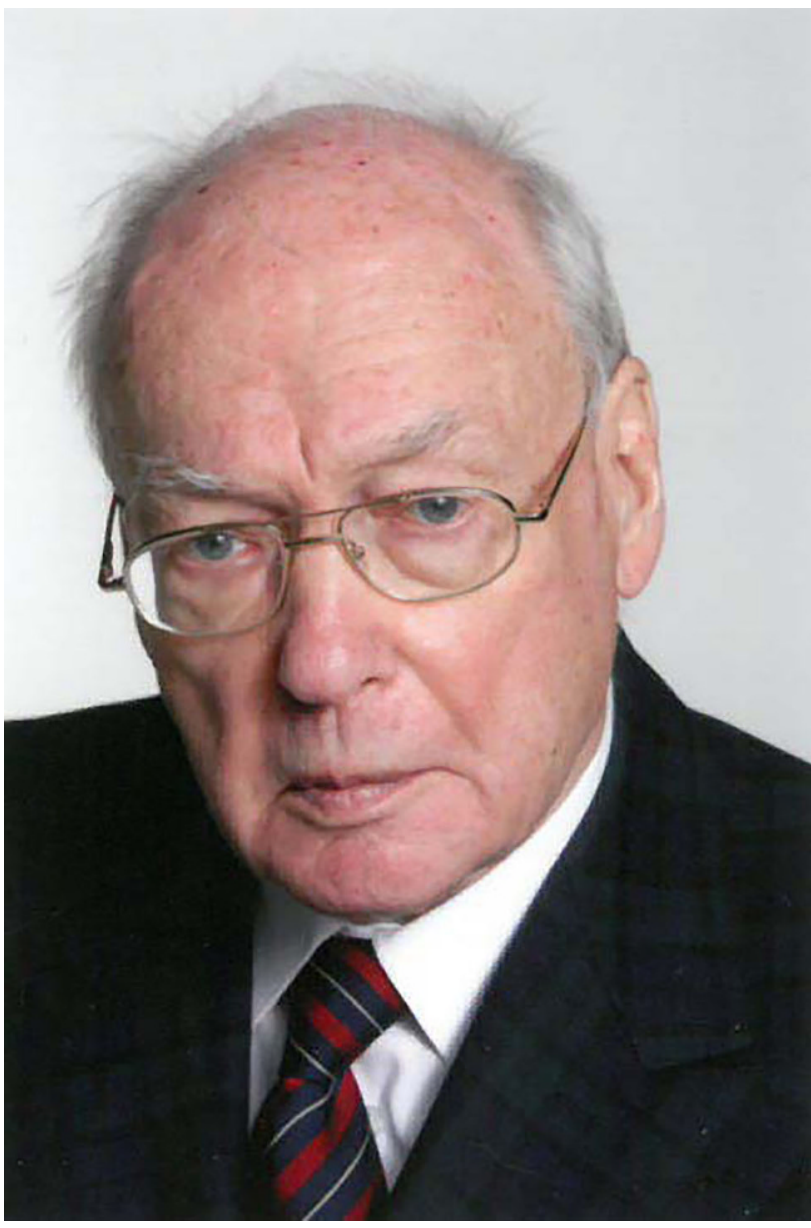

Hellmut Fischmeister wurde 1927 in Wien geboren. Er studierte an der Karl-Franzens-Universität in Graz Physik, Mathematik und Chemie und promovierte 1951 unter Prof. Kratky auf dem Gebiet der Röntgenkristallographie.

\section{B. Hribernik ( $\triangle$ )}

ASMET,

A 8700 Leoben, Österreich

Bruno.Hribernik@asmet.at
Zu Beginn seiner Laufbahn war er in Schweden unter anderem bei der Firma Ericsson und später beim Edelstahlwerk Söderfors tätig. Dort legte er als Forschungschef den Grundstein für die großtechnische Herstellung von Schnellarbeitsstählen aus gasverdüsten Pulvern.

Hellmut Fischmeister war auch in verschiedenen Funktionen an den Universitäten in Uppsala und Stockholm tätig. Er habilitierte sich 1961 an der Universität Uppsala für das Fachgebiet "Allgemeine und Anorganische Chemie..Im Jahr 1965 folgte er einem Ruf an die Chalmers Technische Hochschule in Göteborg und wurde Ordinarius und Vorstand des Instituts für metallische Werkstoffe. Dort setzte er vor allem neue Akzente auf den Gebieten der quantitativen Metallographie, der Pulvermetallurgie, der Deformation zweiphasiger Legierungen und bei der Entwicklung von neuen Hochtemperaturwerkstoffen und metallischen Verbundwerkstoffen.

Im Jahr 1975 trat Hellmut Fischmeister die Nachfolge von Prof. Mitsche als Ordinarius für Metallkunde und Werkstoffprüfung an der Montanuniversität Leoben an. Hier bildeten die Schnellarbeitsstähle und Erstarrungsvorgänge unter der Bedingung der Schwerelosigkeit neue Forschungsschwerpunkte. Als Lehrer etablierte er die Leobener Schule der Werkstoffwissenschaft, welche die physikalisch chemischen Grundlagen der Werkstoffe zum Verständnis ihrer Eigenschaften, ihrer Herstellung und ihrer Anwendung in den Fokus der Lehre stellt. Viele seiner ehemaligen Schüler wurden ebenfalls Universitätsprofessoren und führen diese Tradition noch heute fort.

Von 1981 bis 1994 war Hellmut Fischmeister geschäftsführender Direktor des Max-Planck-Institutes für Metallforschung in Stuttgart, wo er weitere Forschungsthemen aufgriff. Insbesondere waren das die Grenzflächen in Werkstoffen, oxiddispersionsverstärkte Werkstoffe und die hochauflösende Elektronenmikroskopie. Nach dem Mauerfall war er auch von 1991 bis 1993 Gründungsdirektor des MaxPlanck-Institutes (MPI) für Mikrostrukturphysik in Halle. Er wurde 1995 am MPI emeritiert und wirkte anschließend als Honorarprofessor an der TU Graz. 
Aufgrund seiner herausragenden wissenschaftlichen Leistungen erhielt Hellmut Fischmeister auch viele Anerkennungen und Preise, die hier nur auszugsweise dargestellt werden können. So wurde er in fünf Akademien der Wissenschaft gewählt, darunter die Academia Europaea in London und die Österreichische Akademie der Wissenschaften, für die er sich auch nach seiner Emeritierung in vielen Funktionen stark engagiert hat. Er war Ehrenmitglied mehrerer wissenschaftlicher Gesellschaften aus Deutschland, Dänemark, Schweden, Frankreich, den Vereinigten Staaten von Amerika und Japan. Er erhielt Ehrendoktorate der Königlichen Technischen Hochschule in Stockholm, der TU Graz und der Montanuniversität Leoben, war Honorarund Gastprofessor an mehreren Universitäten in Europa und Japan und erhielt hohe Orden, so beispielsweise das Deutsche Bundesverdienstkreuz 1. Klasse.

Darüber hinaus war Hellmut Fischmeister in unzähligen Beratergremien, Beiräten und Aufsichtsräten tätig. So war er auch Berater mehrerer Europäischer Regierungen. Nach seiner Emeritierung konzentrierte er seine Tätigkeiten auf Österreich, wo er weiterhin in der Wissenschaft, Industrie und Politik wichtige Weichenstellungen vorbereitete. Im Erich-Schmid-Institut für Materialwissenschaft der Österreichischen Akademie der Wissenschaften in Leoben war Hellmut Fischmeister als Beirat tätig, beim Materials Center Leoben war er Vorsitzender des Boards. In der Christian Doppler Gesellschaft war es seine Idee, die Laufzeit von Christian Doppler Labororatorien auf max. 7 Jahre zu limitieren. Diese Maßnahme sorgt noch heute für ständig neue Forschungsthemen und für eine ständige Verjüngung der Christian Doppler Forschungsgesellschaft. Von 1994 bis 2006 war Hellmut Fischmeister im Corporate Research Board von Böhler Uddeholm. Er hat dort maßgeblich an der Neuausrichtung und Internationalisierung der Forschung und am Aufbau von Kompetenzzentren in Österreich, Schweden, Deutschland und Brasilien mitgewirkt.

Besonders erwähnenswert sind seine Arbeiten im Österreichischen Universitätenkuratorium von 1993 bis 2002 und im österreichischen Wissenschaftsrat von 2003-2009, welche die wichtigsten fachliche Beratergremien für das Wissenschaftsministerium bilden. Hier hat er die Österreichische Forschungs- und Forschungsförderungslandschaft entscheidend in Hinblick auf Qualitätbewusstsein, internationale Vergleichbarkeit und Transparenz mitgestaltet.

Prof. Hellmut Fischmeister war eine herausragende Forscherpersönlichkeit. Er hat durch seine Arbeiten vor allem im Bereich der Werkstoffwissenschaft das Verständnis um die Entwicklung, Eigenschaften, Herstellung und Anwendung der Werkstoffe entscheidend beeinflusst. Über das rein Fachliche hinaus hatte er großen Einfluss auf Wirtschaft und Gesellschaft. An den Stätten seines Wirkens hat er viele positive Maßnahmen gesetzt, die noch lange nachwirken werden.

Unsere ganze Anteilnahme gilt seiner Ehefrau Ingrid und seiner Familie.

Wir alle werden Hellmut Fischmeister aufrichtig vermissen und ihm ein würdiges Andenken wahren.

Glückauf für Deine letzte Schicht

Horst Cerjak

Robert Danzer

Herbert Danninger

Gerhard Hackl

Bruno Hribernik 